Vietnam Journal of Mechanics, VAST, Vol.41, No. 3 (2019), pp. 233 - 241

DOI: https://doi.org/10.15625/0866-7136/13717

\title{
EFFECTIVE MEDIUM APPROXIMATION FOR CONDUCTIVITY OF COATED-INCLUSION COMPOSITES WITH ANISOTROPIC COATING
}

\author{
Tran Bao Viet ${ }^{1, *}$, Nguyen Thi Huong Giang ${ }^{1}$, Pham Duc Chinh ${ }^{3}$ \\ ${ }^{1}$ University of Transport and Communications, Hanoi, Vietnam \\ ${ }^{2}$ Institute of Mechanics, VAST, Hanoi, Viet Nam \\ *E-mail: viettb@utc.edu.vn
}

Received: 30 March 2019 / Published online: 24 June 2019

\begin{abstract}
Effective medium approximations are constructed in this paper to estimate the macroscopic conductivity of coated-inclusion composites with thin anisotropic coating. The two-phase coated-inclusion are substituted by equivalent one-phase inclusion, using the multi-coated spheres assemblage and the differential substitution approaches. Then, the usual effective medium approximation schemes are applied to the equivalent medium to estimate the conductivity of original three-phase composites. The results obtained were compared with the numerical simulation by finite element method in $2 \mathrm{D}$ show the effectiveness of the methods.
\end{abstract}

Keywords: coated-inclusion; effective conductivity; equivalent-inclusion approach; anisotropic coating.

\section{INTRODUCTION}

A widely recognized observation is that the effective behavior of a matrix-inclusion composites depends on the coating shells (interface or chemical reaction layer). Over several decades, determining the thermal gradient and flux fields in the layers has become a interesting subject for numerous theoretical [1-9].

Simple analytical approaches are developed recently by us to estimate macroscopic properties of coated-inclusion composites [10-14]. However, these studies only mentioned the case of isotropic coating. This paper is concerned with the determination of the effective conductivity of coated-inclusion composite with thin anisotropic coating by simple analytical approach. The two-phase coated-inclusion is substituted by equivalent one-phase inclusion, using the multi-coated spheres assemblage and the differential substitution approaches. Then, the usual effective medium approximation schemes are applied to the equivalent medium to estimate the conductivity of original three-phase composites. The results obtained were compared with the numerical simulation by finite element method in 2D to show the effectiveness of the methods.

(C) 2019 Vietnam Academy of Science and Technology 


\section{THEORETICAL HOMOGENIZATION FRAMEWORK}

\subsection{The sphere assemblage model of two phase material}

We start with a particularly simple situation where the two component $d$-dimensional composite is a suspension of random spherical/circular inclusions of conductivity $c_{1}$ and volume proportion $v_{1}$ in a continuous matrix of conductivity $c_{M}$ and volume fraction $v_{M}$. The main idea of the sphere assemblage model of two phase matrix-based material is that we consider a spherical/circular inclusion surrounded by a coated spherical/circular matrix shell embedded in an effective equivalent infinite medium (Fig. 1). The effective conductivity of the composite is calculated based on the Hashin-Strickman twophase coated spheres assemblage and Hill substitution scheme [15]

$$
c^{e f f}=P\left(v_{1}, c_{1}, c_{M}\right)=\left(\frac{v_{1}}{c_{1}+(d-1) c_{M}}+\frac{v_{M}}{d c_{M}}\right)^{-1}-(d-1) c_{M} .
$$

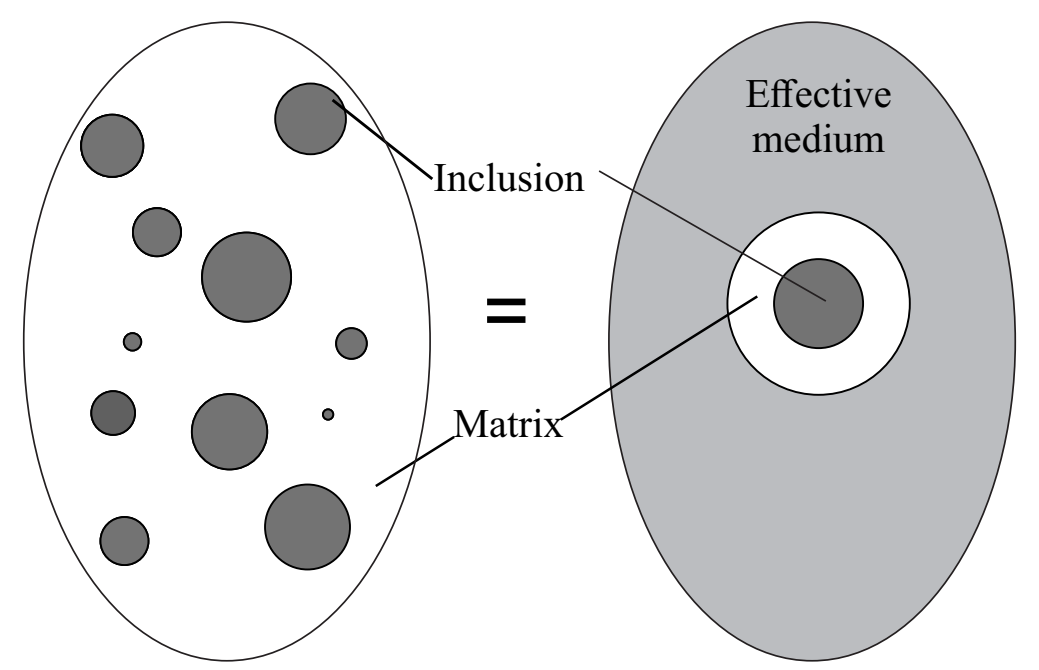

Fig. 1. the sphere assemblage model of two phase material

Two consequences of (1) corresponding respectively with the case of $v_{1} \rightarrow 0$ and the opposite case of $v_{M} \rightarrow 0$ are respectively

$$
c^{e f f}=c_{M}+v_{1} \frac{\left(c_{1}-c_{M}\right) d c_{M}}{c_{1}+(d-1) c_{M}}+O\left(v_{1}^{2}\right),
$$

and

$$
c^{e f f}=c_{1}+v_{M} \frac{\left(c_{M}-c_{1}\right)\left[c_{1}+(d-1) c_{M}\right]}{d c_{M}}+O\left(v_{M}^{2}\right) .
$$

It is necessary to note that Eq. (2) are the theoretical dilute solution results for the inhomogeneities suspended in an infinite matrix while Eq. (3) present the effective conductivity of the suspension of thin coating inclusion that is used below for further calculations in this paper. The two effective conductivities from $(2,3)$ obey the Hashin-Shtrikman 
bounds which are the best mathematical bounds based on the component properties and volume content of $d$-dimensional composites,

$$
\mathrm{HSL}=P\left(c_{\min }\right) \leq c^{e f f} \leq P\left(c_{\max }\right)=\mathrm{HSU},
$$

with

$$
c_{\min }=\min \left\{c_{M}, c_{1}\right\}, \quad c_{\max }=\max \left\{c_{M}, c_{1}\right\},
$$

and

$$
P(c)=\left(\frac{v_{M}}{c_{M}+c_{*}}+\frac{v_{1}}{c_{1}+c_{*}}\right)^{-1}-c_{*}, \quad c_{*}=(d-1) c_{1} .
$$

\subsection{Differential substitution construction}

Now we consider a more complex situation where the inclusion characterizing by $c_{1}, v_{1}$ surrounded by a thing coating shell of conductivity $c_{c}$ and volume fraction $v_{c}$. To account for the thin coating effect, we base on the differential scheme construction process proposed recently in Pham et al. [16]. In which, Pham consider that the thin coating shell is divided into some infinitesimal volume amounts $\Delta v$ of spherical coating shell of radially variable conductivity $c_{c}(r)$ with $r$ is radius from the shell to the center of inclusion (in this paper we consider that $c_{\mathcal{c}}(r)={c_{c}}_{c}$ ). By combining Eq. (3) and the differential substitution procedure (in a similar way as the classical differential scheme), the equivalent conductivity of the thin coated inclusion can be obtained from the differential equation

$$
\frac{\mathrm{d} c}{\mathrm{~d} v}=\frac{1}{1-v} \frac{\left(c_{c}-c\right)\left[c+(d-1) c_{c}\right]}{d c_{c}}, \quad c(v=0)=c_{1}, \quad c^{1 c}=c\left(v=v_{c}\right) .
$$

Then we replace the inclusion $\left(c_{1}, v_{1}\right)$ by the coated inclusion having the effective conductivity $c^{1 c}$ and volume proportion $v_{1 c}$ in Eqs. (1)-(3), we obtain the respective effective conductivity formulas of the matrix-based composite materials with coated inclusions.

\section{THIN ANISOTROPIC COATINGS WITH RADIALLY VARIABLE CONDUCTIVITIES AND EQUIVALENT INCLUSION APPROACH}

In the section, we are interested in constructing a simple approximation to take into account the effect of thin anisotropic coatings on the effective conductivity of the suspension of the coated inclusions in the matrix. To do this, the composite material is composed of the spherical inclusions $V_{1}$ of radius $R_{1}$, volume proportion $v_{1}$, and isotropic conductivity $c_{1}$, is coated by the spherical shell $V_{c} \backslash V_{1}$ of outer radius $R_{c}$ volume proportion $v_{c}$, and anisotropic conductivity, with the normal (in the radial direction) conductivity $c_{N}$ and transverse (in the coating surface directions) conductivity $c_{T}$. The coated sphere then is embedded in the matrix shell $V_{M} \backslash V_{1}$ of outer radius $R_{M}$, volume proportion $v_{M}$, and isotropic conductivity $c_{M}$. The anisotropic shell can be equivalently presented as being composed of $2 m$ ultra-thin spherical shell coatings of thickness $\frac{h}{2 m}=\frac{R_{c}-R_{1}}{2 m}$, and 
isotropic conductivities $c_{2}$ and $c_{3}$, alternately, in the limit $m \rightarrow \infty$, with

$$
c_{T}=\frac{1}{2}\left(c_{2}+c_{3}\right), \quad c_{N}=2\left(c_{2}^{-1}+c_{3}^{-1}\right)^{-1},
$$

while

$$
v_{2}=v_{3}=v_{c} / 2 \text {. }
$$

Following the mathematical developments presented above, we have a asymptotic expression

$$
\begin{aligned}
c^{1 c} & =c_{1}+\frac{\Delta v}{2 m}\left(m \frac{\left(c_{2}-c_{1}\right)\left[c_{1}+(d-1) c_{2}\right]}{d c_{2}}+m \frac{\left(c_{3}-c_{1}\right)\left[c_{3}+(d-1) c_{1}\right]}{d c_{3}}\right)+O\left(c_{c}^{2}\right) \\
& =c_{1}+\frac{\Delta v}{d c_{N}}\left(c_{I}-c_{1}\right)\left(c_{1}+c_{I I}\right)+O\left(c_{c}^{2}\right),
\end{aligned}
$$

where

$$
\begin{aligned}
c_{I} & =\frac{1}{2}\left\{\left[(d-2)^{2} c_{N}^{2}+4(d-1) c_{T} c_{N}\right]^{1 / 2}-(d-2) c_{N}\right\}, \\
c_{I I} & =\frac{1}{2}\left\{\left[(d-2)^{2} c_{N}^{2}+4(d-1) c_{T} c_{N}\right]^{1 / 2}+(d-2) c_{N}\right\} .
\end{aligned}
$$

Letting $m \rightarrow \infty$, we obtain the ordinary differential equation determining the effective conductivity of the assemblage of coated inclusions, with inclusions having conductivity $c_{1}$, volume proportion $v_{1}$, and anisotropic coating of variable conductivities $c_{N}(v)$, $\mathcal{c}_{T}(v)$, volume proportion $v_{\mathcal{C}}$

$$
\frac{\mathrm{d} c}{\mathrm{~d} v}=\frac{1}{1-v} \frac{\left(c_{I}-c\right)\left(c+c_{I I}\right)}{d c_{N}}, \quad c(0)=c_{1}, \quad c^{\text {eff }}=c\left(v_{c}\right) .
$$

In the case $c_{N}=$ const, $c_{T}=$ const, Eq. (12) can be integrated explicitly

$$
c^{1 c}=c^{e q}=\frac{c_{I I}\left(c_{1}-c_{I}\right)+c_{I}\left(c_{1}+c_{I I}\right) \tilde{v}_{1}^{\frac{d c_{N}}{c_{I}+c_{I I}}}}{c_{I}-c_{1}+\left(c_{1}+c_{I I}\right) \tilde{v}_{1}^{\frac{d c_{N}}{c_{I}+c_{I I}}}}, \quad \tilde{v}_{1}=\frac{v_{1}}{v_{1}+v_{c}}, \quad v^{e q}=v_{1}+v_{c} \text {. }
$$

From the formula (13) for the effective conductivity of the assemblage of coated inclusions with inclusions having anisotropic coating of variable conductivities, we propose a hypothesis that the real coated inclusion is replaced by a fictive equivalent and homogeneous inclusion with the volume factor noted by $v^{e q}=v_{1}+v_{c}$ and $c^{e q}$ having the value from the formula (15). One the coated inclusion is replaced by the homogeneous inclusion, the effective conductivity of the original material can be obtained by the classical effective medium approximations. According to (1), the coated inclusion composite has the effective conductivity

$$
c^{e f f}=\left(\frac{v^{e q}}{c^{e q}+(d-1) c_{M}}+\frac{v_{M}}{d c_{M}}\right)^{-1}-(d-1) c_{M} .
$$

In the general situation where the material is composed of the matrix and the different type of inclusions with anisotropic coating layer, the equivalent strategy is taken 
into account for all different type of inclusions then we have a multicomponent composite material with different type of equivalent inclusions having conductivity $c^{e q 1}$, volume fraction $v^{e q 1}$; conductivity $c^{e q 2}$, volume fraction $v^{e q 2} ; \ldots ; c^{e q \beta}$, volume fraction $v^{e q \beta}$ in a matrix of conductivity $c_{M}$, volume fraction $v_{M}$. It necessary to note that $c^{e q \beta}$ can be also calculated by (13). Then the effective conductivity of the multicomponent matrix-based composite can be determined by applying the simple polarization approximation [14]

$$
c^{e f f}=\left(\sum_{\beta} \frac{v^{e q \beta}}{c^{e q \beta}+(d-1) c_{M}}+\frac{v_{M}}{d c_{M}}\right)^{-1}-(d-1) c_{M} .
$$

\section{NUMERICAL SIMULATIONS AND APPLICATIONS}

In order to verify the above result, we make finite element calculations for a number of periodic suspensions of circles in two dimensions. Due to the periodicity condition of the microscopic heat flux field $q(\underline{z})$, the average of the microscopic heat flux fields $q(\underline{z})$ over the domain of periodic cell $\mathcal{U}$ and the Representative Volume Element $V$ are equal. This indicates that the macroscopic relationships can be determined numerically from the solution over the finite domain $\mathcal{U}$. Some details concerning the global temperature field equations, the boundary conditions, the open source finite element code used .... are identical than the ones presented in works of Tran et al. [14], and no need to rewrite in this text. The improvement of numerical simulation in this paper come from the anisotropic properties of the coating shell. In which, two types of rectangular unit cell are accounted for calculation (square and hexagonal arrays of coated circles where their lengths are denoted by $a_{1}$ and $a_{2}$ - see Fig. 2). To model anisotropic coatings with radially variable conductivities $c_{N}$ and $c_{T}$, we divide the coated shell into some parts of same size, shape and different direction characterizing by angular $\beta$ and $\alpha$ (Fig. 3). For each part (characterizing by $\alpha$ and $\beta$ ), conductivities are fixed at $\mathbf{c}\left(c_{11}, c_{22}, c_{21}\right)$ in the global coordinate $\left(x_{1}, x_{2}\right)$ depend on $c_{N}, c_{T}$ and position point that define by local coordinate $\left(x_{1}^{\prime}, x_{2}^{\prime}\right)$ (Fig. 3$)$

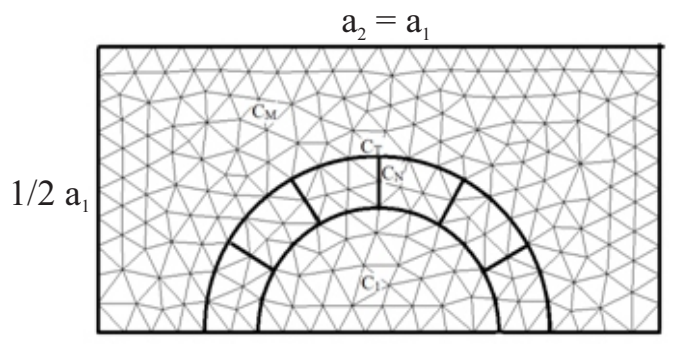

(a)

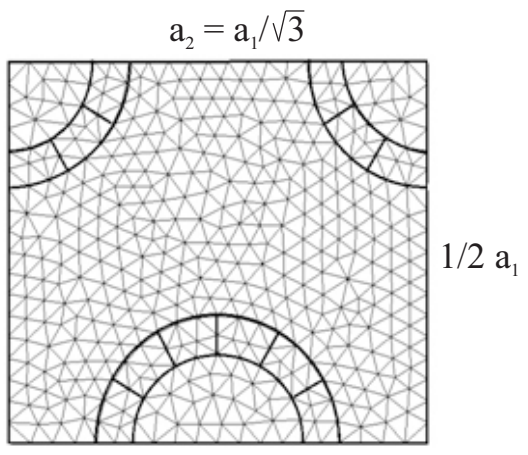

(b)

Fig. 2. Periodic cell: (a) - square array; (b) - hexagonal array 
by the relationships

$$
\begin{aligned}
& c_{11}=c_{N} \cos ^{2} \alpha+c_{T} \sin ^{2} \alpha, \\
& c_{22}=c_{N} \sin ^{2} \alpha+c_{T} \cos ^{2} \alpha, \\
& c_{21}=\left(c_{N}-c_{T}\right) \cos \alpha \sin \alpha .
\end{aligned}
$$

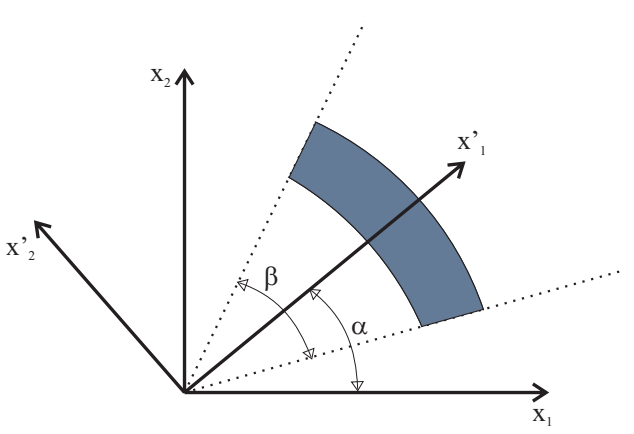

Fig. 3. Rotational coordinate transformation

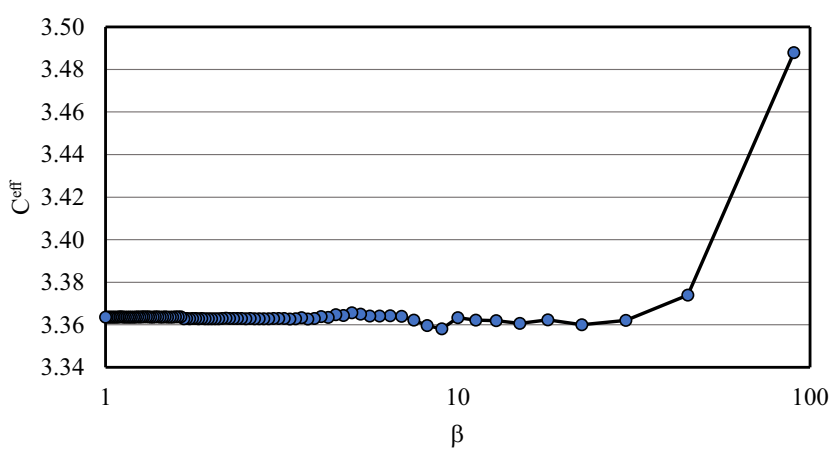

Fig. 4. Angular convergence test

In fact, the angle $\beta$ need enough small to guarantee the homogeneous properties of materials. A Finite element method convergence test between angular value and effective conductivity are presented in Fig. 4 with $c_{M}=1, c_{1}=100, c_{T}=50, c_{N}=30, v_{1}=10 v_{c}$, $v_{1 c}=v^{e q}=0.5$. From this test, we adopt a value of $\beta=3^{o}$ for the further numerical calculations.

For particular calculations, we take $c_{M}=1, c_{1}=100, c_{T}=50, c_{N}=30$ (and $\left.c_{M}=100, c_{1}=1, c_{T}=70, c_{N}=50\right), v_{1}=10 v_{c}, v_{1 c}=v^{e q}=v_{1}+v_{c}=0 \rightarrow 0.78$ for square array of coated circles and $v_{1 c}=v_{1}+v_{c}=0 \rightarrow 0.905$ for the hexagonal array. The curves in Figs. 5 and 6 show that the numerical calculations for both equivalent and original medium are close for all the ranges of parameters up to the maximal packing of the circles, even though the component properties differ largely. In Figs. 5 and 6, the MoriTanaka approximation that coincide with Hashin-Shtrikman bounds and the polarization approximation (14), the dilute approximation for the equivalent homogeneous-inclusion composite (2) are also compared.

In next examples, we account for the influence of the ratio $c_{T} / c_{N}(1 \rightarrow 6)$ to the effective conductivity of the suspension. The composite is composed of a continuous matrix with $c_{M}=1$, and by coated anisotropic circular inclusions with $c_{1}=100, c_{N}=10$. We fix also $v_{1}=10 v_{c}$ and $v_{1 c}=0.5$. Numerical configurations considered are square and hexagonal array of coated circles and equivalent homogeneous circles. Fig. 7 presents respectively some numerical results and analytical estimates for square and hexagonal arrays. Fig. 8 is the same as in Fig. 7 with $c_{M}=100, c_{1}=1$. In these situations, the Mori-Tanaka approximation (14) appears good regarding its simplicity and generality. 


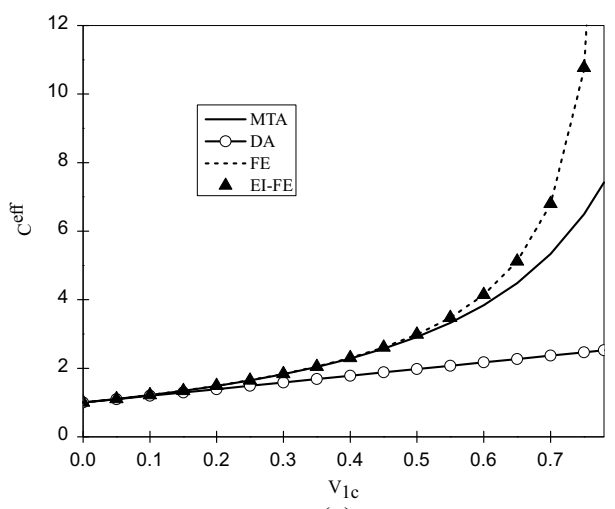

(a)

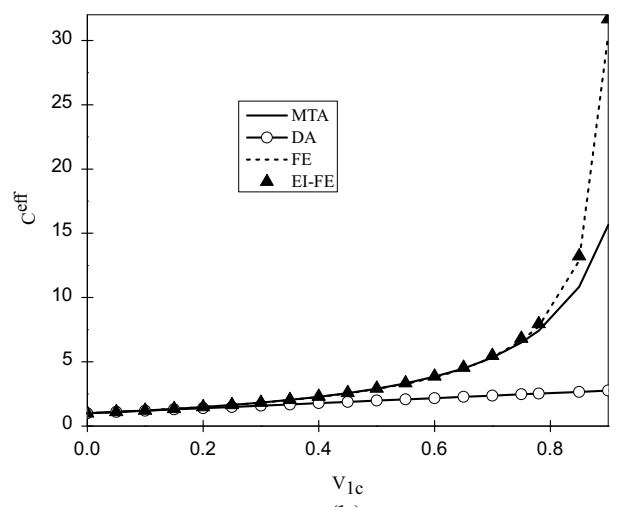

(b)

Fig. 5. Effective conductivity of array of circles ((a)-square; (b)-hexagonal) with $c_{M}=1, c_{1}=$ $100, c_{T}=50, c_{N}=30$; FE - Finite element numerical result; EI-FE - Finite element results for the equivalent homogeneous-inclusion composite; DA - dilute approximation; MTA-Mori-Tanaka approximation
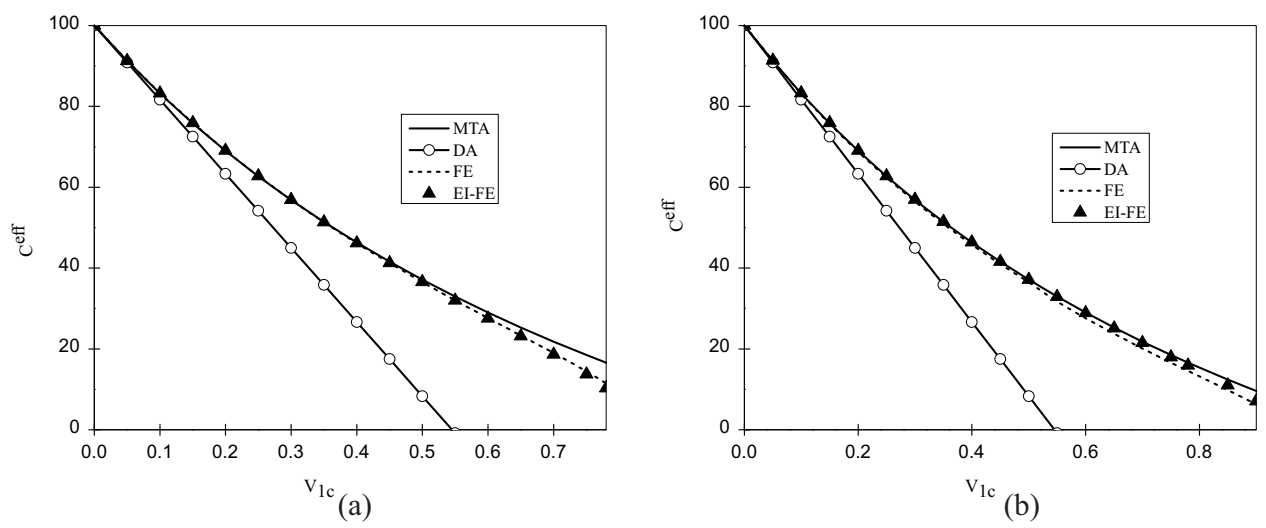

Fig. 6. The same as in Fig. 5 of array of circles with $c_{M}=100, c_{1}=1, c_{T}=70, c_{N}=50$

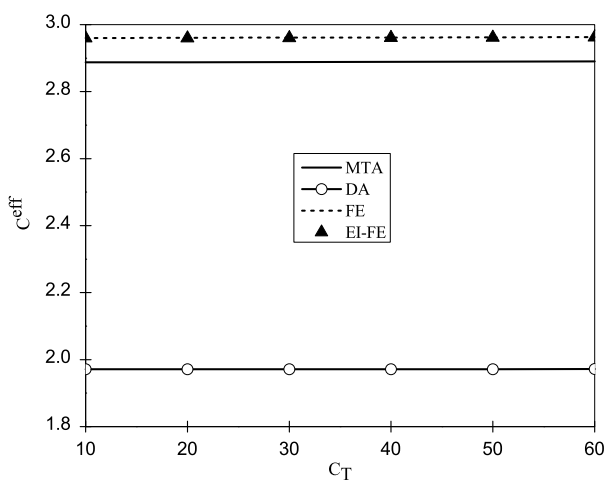

(a)

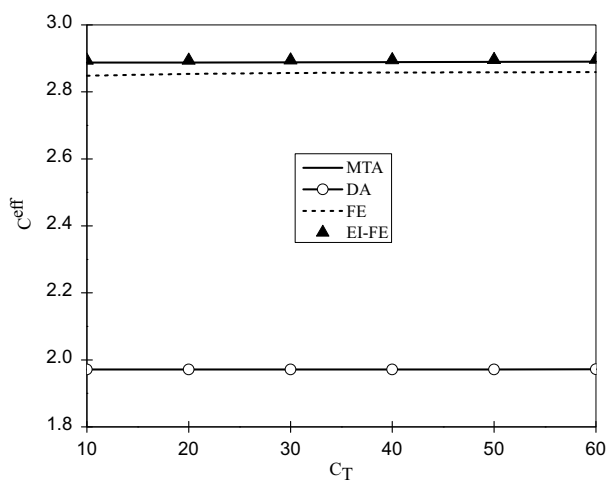

(b)

Fig. 7. The same as in Fig. 5 of array of circles with $c_{M}=1, c_{1}=100, c_{N}=10, v_{1 c}=0.5$ 


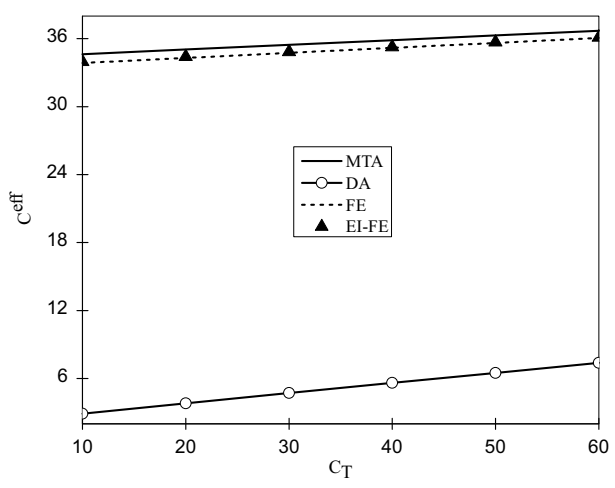

(a)

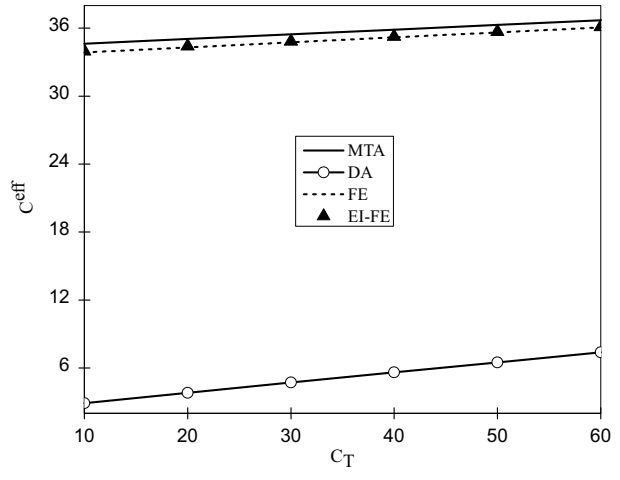

(b)

Fig. 8. The same as in Fig. 5 of array of circles with $c_{M}=100, c_{1}=1, c_{N}=10, v_{1 c}=0.5$

\section{CONCLUSIONS}

Based on the multi-coated spheres assemblage and the differential substitution approaches at dilute configuration, the two-phase coated-inclusion with thin anisotropic coating are substituted by equivalent one-phase inclusion. Then, the polarization approximation that coincide with well-know Mori-Tanaka approximation in the case of coated circle inclusions are applied to determine the the conductivity of original composites. The results obtained were compared with the numerical simulation by finite element method in 2D. The comparison has shown the effectiveness of the methods. This strategy presented in the paper is a novel and simple method to account the influence of the anisotrop coating to the global conductivity of multicomponent matrix-based composite material.

Developments of the approximations to the cases of anisotropic particle distribution, more complex material structure and those involving the effect of aggregate size distribution are interesting subjects for the further studies.

\section{ACKNOWLEDGMENT}

This research is funded by Vietnam National Foundation for Science and Technology Development (NAFOSTED) under grant number 107.02-2018.306.

\section{REFERENCES}

[1] E. Hervé and A. Zaoui. Elastic behaviour of multiply coated fibre-reinforced composites. International Journal of Engineering Science, 33, (10), (1995), pp. 1419-1433. https://doi.org/10.1016/0020-7225(95)00008-1.

[2] Y. P. Qiu and G. J. Weng. Elastic moduli of thickly coated particle and fiberreinforced composites. Journal of Applied Mechanics, 58, (2), (1991), pp. 388-398. https://doi.org/10.1115/1.2897198.

[3] C.-W. Nan, R. Birringer, D. R. Clarke, and H. Gleiter. Effective thermal conductivity of particulate composites with interfacial thermal resistance. Journal of Applied Physics, 81, (10), (1997), pp. 6692-6699. https://doi.org/10.1063/1.365209. 
[4] H. Le Quang, D. C. Pham, G. Bonnet, and Q.-C. He. Estimations of the effective conductivity of anisotropic multiphase composites with imperfect interfaces. International Journal of Heat and Mass Transfer, 58, (1-2), (2013), pp. 175-187. https://doi.org/10.1016/j.ijheatmasstransfer.2012.11.028.

[5] I. V. Andrianov, A. L. Kalamkarov, and G. A. Starushenko. Three-phase model for a fiber-reinforced composite material. Composite Structures, 95, (2013), pp. 95-104. https://doi.org/10.1016/j.compstruct.2012.07.003.

[6] M. Hori and S. Nemat-Nasser. Double-inclusion model and overall moduli of multi-phase composites. Mechanics of Materials, 14, (3), (1993), pp. 189-206. https://doi.org/10.1115/1.2904292.

[7] D. S. Liu and D. Y. Chiou. Modeling of inclusions with interphases in heterogeneous material using the infinite element method. Computational Materials Science, 31, (3-4), (2004), pp. 405420. https://doi.org/10.1016/j.commatsci.2004.05.002.

[8] Y. M. Shabana. A micromechanical model for composites containing multi-layered interphases. Composite Structures, 101, (2013), pp. 265-273. https://doi.org/10.1016/j.compstruct.2013.02.008.

[9] P. D. Chinh and T. A. Binh. Equivalent-inclusion approach for the conductivity of isotropic matrix composites with anisotropic inclusions. Vietnam Journal of Mechanics, 38, (4), (2016), pp. 239-248. https://doi.org/10.15625/0866-7136/6753.

[10] T. K. Nguyen and D. C. Pham. Equivalent-inclusion approach and effective medium estimates for elastic moduli of two-dimensional suspensions of compound inclusions. Philosophical Magazine, 94, (36), (2014), pp. 4138-4156. https://doi.org/10.1080/14786435.2014.978831.

[11] D. C. Pham and B. V. Tran. Equivalent-inclusion approach and effective medium approximations for conductivity of coated-inclusion composites. European Journal of Mechanics-A/Solids, 47, (2014), pp. 341-348. https://doi.org/10.1016/j.euromechsol.2014.05.010.

[12] B. V. Tran, D. C. Pham, and T. H. G. Nguyen. Equivalent-inclusion approach and effective medium approximations for elastic moduli of compound-inclusion composites. Archive of Applied Mechanics, 85, (12), (2015), pp. 1983-1995. https://doi.org/10.1007/s00419-015-10316.

[13] T. N. Quyet, P. D. Chinh, and T. A. Binh. Equivalent-inclusion approach for estimating the elastic moduli of matrix composites with non-circular inclusions. Vietnam Journal of Mechanics, 37, (2), (2015), pp. 123-132. https://doi.org/10.15625/0866-7136/37/2/5659.

[14] B.-V. Tran, D.-C. Pham, and T.-H.-G. Nguyen. Effective medium approximation for conductivity of unidirectional coated-fiber composites. Computational Thermal Sciences: An International Journal, 9, (1), (2017), pp. 63-76. https://doi.org/10.1615/computthermalscien.2017015911.

[15] Z. Hashin and S. Shtrikman. A variational approach to the theory of the effective magnetic permeability of multiphase materials. Journal of Applied Physics, 33, (10), (1962), pp. 31253131. https://doi.org/10.1063/1.1728579.

[16] D. C. Pham. Solutions for the conductivity of multi-coated spheres and spherically symmetric inclusion problems. Zeitschrift für angewandte Mathematik und Physik, 69, (1), (2018), p. 13. https://doi.org/10.1007/s00033-017-0905-6. 
